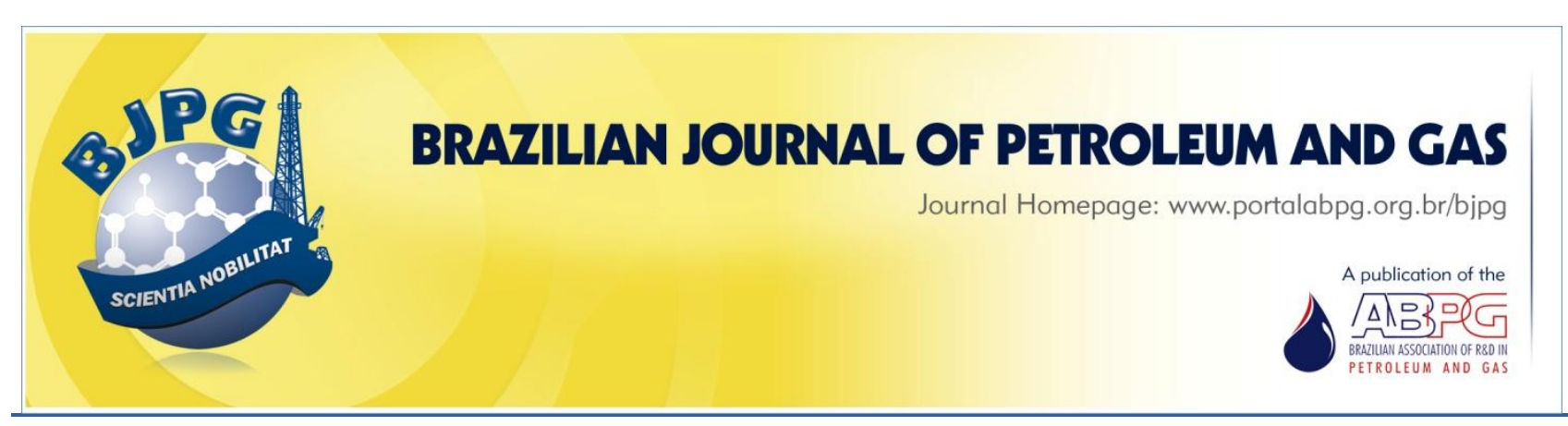

\title{
A NEW SIMULATION MODEL FOR TWO-PHASE FLOW IN POROUS MEDIA
}

\author{
${ }^{\text {a }}$ Idorenyin, E.; ${ }^{\text {a }}$ Shirif, E. ${ }^{1}$ \\ a University of Regina, Faculty of Engineering and Applied Science, Petroleum Systems Engineering, Regina, SK, Canada
}

\begin{abstract}
Oil production from petroleum reservoirs is a result of its displacement from the reservoir pore space by either gas and/or water. The displacement process is simulated using Darcy's law as the basis of an axiomatic transport rule. However, Darcy's law is a phenomenologically derived constitutive equation that describes flow in porous media from a macroscopic standpoint. It fails to account for microscopic fluid interactions and pore-level variations that play significant roles even in the macroscopic manifestation of multiphase flow. This study presents a novel mathematical model to describe pore-level displacement processes in porous media. The flow medium considered in this work is a representative "physical model" made of parallel uniform circular capillary tubes, assembled to form a porous structure, which allows cross-flow from one tube to the other.
\end{abstract}

\section{KEYWORDS}

two-phase flow; circular flow; immiscible flow; simulation; capillary tubes

\footnotetext{
${ }^{1}$ To whom all correspondence should be addressed.

Address: University of Regina, Faculty of Engineering and Applied Science, Petroleum System Engineering, Regina, Saskatchewan

Telephone / Fax: +1 306-585-4955 / +1 306-337-2620| E-mail: ezeddin.shirif@uregina.ca doi:10.5419/bjpg2012-0001
} 


\section{INTRODUCTION}

From reservoir engineering perspective, the field of multiphase flow embraces the motion of immiscible/miscible fluids (oil, water and gas) through the voids of a porous domain. The fluid phases are chemically unreactive. In this sort of fluid transport, the fluids flow together, allowing a distinct interface between them. A typical example is observed during secondary oil recovery (such as water flooding and gas injection) from petroleum reservoirs in which the production of one fluid phase occurs as a direct result of the displacement of that fluid phase from the reservoir pores by another fluid phase. It is the goal of reservoir engineers to study and understand fluid transport in petroleum reservoirs and to use the information acquired to forecast, manage and control petroleum production. This kind of study often involves building simulation models that can mimic actual fluid flow behaviours in petroleum reservoirs. The models can be considered as an analogous representation of the porous medium (the reservoir), with all the appropriate physics, reservoir properties, and geology being matched as closely as possible.

Fluid transport in porous media appears to be solvable in two alternative approaches, based upon either the scale of macroscopic systems or the scale of pore dimensions. The macroscopic approach is the method employed in traditional reservoir simulation. In this approach, the fluid flow is described from the standpoint of aggregate reservoir properties, that is, quantities are averaged over many pores in different lump sections (grid blocks) of the reservoir. The porous medium is discretized spatially into cells, with each cell being assigned a representative average value of each reservoir property. A second discretization in time is also imposed on the system. With appropriate initial and boundary conditions, the solution to the flow problem is obtained progressively in each cell and at each time step. However, it is important to point out that the rock properties contained in the flow problem are obtained from empirical correlations. The macroscopic approach assumes the phenomenological Darcy's law, which contains an "undetermined" constant called permeability as the basis of an axiomatic transport rule in porous media. Empirical relationships, in this wise, are not always sufficient to account fully for the physics of flow, especially at scales larger than laboratory cores. As a result, the macroscopic approach fails to correctly account for all the intrinsic controlling factors (including pore-level variations in fluid flow and microscopic fluid interactions) that interplay in a multiphase displacement process.

To address the inherent limitations contained in the macroscopic approach, many researchers have attempted to solve the problem of fluid flow in porous media using an entirely different approach, based on microscopic pore dimension. These researchers incorporated physicochemical properties, such as wettability, contact angle, and interfacial tension, in their formulation of porescale models. In principle, these pore-scale models lead to solutions from which important deductions could be made. Pore-level studies have proven to be capable of providing useful insights in the understanding of even macroscopic manifestation of fluid flow mechanisms in porous media.

The pore structure of the vast majority of porous media consists of a three-dimensional system of voids, pores, or capillaries which, on one hand, provide storage space and, on the other hand, serve as flow paths when the voids are interconnected. Several attempts have been made to construct representative analogs of porous structure upon which to base flow simulation. Of all the pore-scale models that have evolved, three lend themselves to the greater prospect of being very useful in the study of fluid flow in porous media: the network model, the sphere-pack model, and the tube-bundle model. The use of the network model in simulating flow phenomena in porous media was introduced by Fatt (1956). Network models represent the void space of a reservoir rock by a lattice of bonds and nodes. Many of the latter proposed network models are variants of Fatt's model in terms of connectivity, shape of pore segments and pore-level physics (Dullein, 1975; Dias and Payatakes, 1986; Blunt and King, 1991; Øren and Bakke, 1998, Patzek, 2001). The disadvantage of using network simulation is that a model of, say, a thin slice of a porous medium involves hundreds of thousands of equations, which place a heavy demand on computational resources and time.

The sphere-pack model simplifies pore structures to a collection of spheres with void spaces between them. The packing could be either 
regular, with cubic, orthorhombic, rhombohedral or hexagonal arrays, or intermediate, often assumed as a mixture of two or more arrays. Although the sphere-pack model can be used for almost any transport phenomena in porous media, the complexity of the pore geometry so formed prevents the derivation of accurate equations of flow. As a result, the model yields equations that can lead to misrepresentation of data. Agreement between theory and observation has been achieved for this model by inserting parameters of doubtful physical significance. For example, Rapoport and Leas (1951) have attempted to derive a relationship between capillary pressure and the permeability of the wetting phase using the Carman-Kozeny equation (Kozeny, 1927; Carman, 1937 and 1956). However, they made an assumption in their treatment of the problem that entirely changed the physical model they set out to address into a bundle of tubes.

The earliest tube-bundle models represent porous media as an assemblage of independent, uniform circular capillary tubes (Childs and CollisGeorge, 1948; Purcell, 1949; Gates and Leitz, 1950; Fatt and Dykstra, 1951). The models produce equations which describe the flow properties of porous media by using relatively tractable mathematical relationships. While some models picture porous media as a system of parallel straight or tortuous tubes (Scott and Dullein, 1962; Rothfeld, 1963), others assume entirely different arrangements. To replicate the pore size distribution of real porous matrices, as well as introduce isotropy into tube-bundle models, various tube configurations have been adopted (e.g. by arranging tubes of different radii in parallel, by varying the diameter of the tubes at random intervals, or by periodically constricting the tubes). Pore size distributions are normally obtained from a relationship of capillary pressure versus saturation data. Two major classes of tube-bundle models exist: the non-interacting and the interacting tube-bundle models. Within each class, several ingenious variations of tube shape and arrangements are possible including parallel tubes, serial tubes, periodically constricted tubes, straight tubes, tortuous tubes, and random adjacent slices of tubes (Brakel, 1974). The non-interacting tubebundle model visualizes a porous medium as an assemblage of independent capillary tubes (Bathley and Ruth, 1999 and 2001). Fluids in adjacent tubes do not interact: there is no cross- flow of fluid between flow channels. A recognized disadvantage of the non-interacting tube-bundle model, arising from flow independence in adjacent tubes, is that fluid-fluid interface travels faster and further in wider tubes, a prediction that is completely antithetical to the observed imbibitions process in porous media (Dong et al., 1998). Recently, the idea of cross-flow was incorporated into the tube-bundle model by Dong et al. (1998, 2005, and 2006). This new proposition assumes ideal connections between adjacent tubes, thus allowing fluid transfer between tubes and, hence, pressure equilibration at all cross sections where identical fluids are present. They used the model to analyze the displacement of a non-wetting fluid by a wetting fluid at different injection rates and arrived at the conclusion that the saturation profiles predicted by this model are consistent with both numerical simulation and experimental results for porous media. The downside of their model is that each of the resulting $n$ evolution equations contains $n$ unknowns, where $n$ corresponds to the number of tubes comprising the porous structure. A greater computational resource is unavoidably required in determining the unknowns.

In this paper, two-phase displacement in porous media is investigated from an objective consideration of the physical reality of fluid flow in a defined pore-scale model called the interacting tube bundle. In itself, this physical model is regarded as an "ideal" sample of a porous medium within which fluid-fluid displacement interfaces in the flow channels are tracked in the progression of time. A new simulation model is proposed with which to investigate two-phase immiscible displacement in the interacting tube bundle. The model produces relatively simpler $n$ evolution equations, each of which contains only three unknowns and, as a result, calls for a shorter computational time.

\section{THE NEW SIMULATION MODEL}

A core sample of a porous medium is replete with hundreds of thousands of connected pore spaces which together provide innumerable channels responsible for fluid transmission. During an immiscible displacement in such a porous system, and due to the interconnection of pores, it 


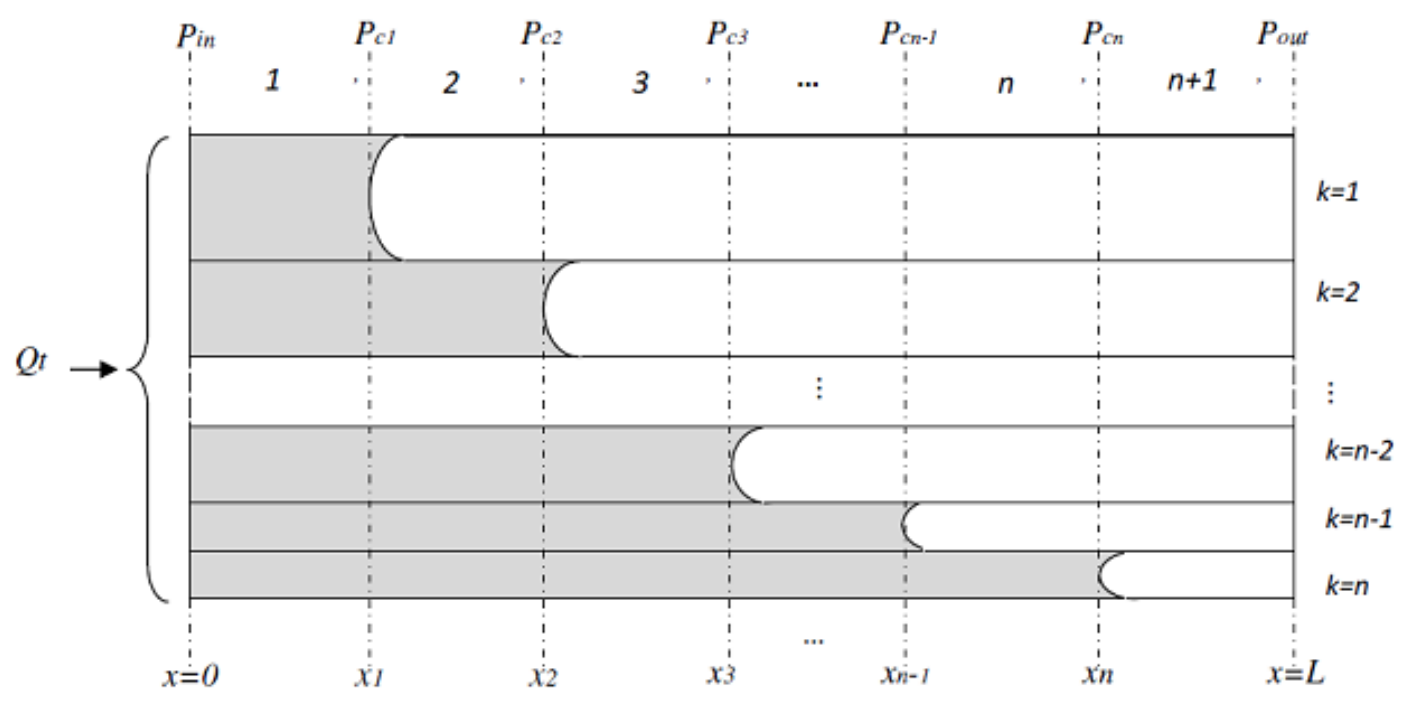

Figure 1. Schematic of interacting tube-bundle model.

is possible for fluid to transfer from one flow channel to another. This phenomenon of crossflow results in pressure equilibration at every perpendicular cross section where the same fluid is flowing past. The interacting tube-bundle model is, in its simplest form, an arrangement of parallel capillary tubes that permits transfer flow; the communication between adjacent tubes. Such a system of flow channels serves as an ideal model with which to study transport process in porous media.

An interacting tube-bundle model comprised of $n$ circular tubes with different radii $R_{k}(k=$ $1,2, \ldots, n$ and $R_{1} \geq R_{2} \geq \cdots \geq R_{n}$ ) but of equal length $L$ (Figure 1 ) will be examined. The following simplifying assumptions are made in the development of the model:

1. The fluids in the porous system are Newtonian and incompressible.

2. The fluids are non-reactive.

3. The flow process occurs under isothermal conditions.

4. The porous system is isotropic.

5. Perfect displacement is assumed in the $Q_{o m}=\frac{\left(P_{o, \mathrm{~m}-1}-P_{o m}\right)}{\left(x_{m}-x_{m-1}\right)} \sum_{k=1}^{m-1} \lambda_{o k}$ model.
Irreducible wetting-phase saturation and residual wetting-phase saturation are not taken into consideration. Assuming that all the tubes are initially filled with oil, the non-wetting phase, the left end of the model is connected to a reservoir of water, the wetting phase, supplying a flow rate of $Q_{t}$ while the right end is connected to an oil receptacle. Water imbibes into the left end of the model and travels further into the smaller tubes. With continued injection, a point is reached when all the tubes are invaded by water, and an oil-water interface is therefore present in each tube. However, the position of the interface in each tube is dependent on those in the adjacent tubes because of transfer flow and pressure equilibration.

The model is split into regions labelled $m=1,2, \ldots, n+1$ (as shown in Figure 1 ) based on the positions of the oil-water interfaces in the tubes. The water and oil flow rates in region $m$ are given, respectively, by the Hagen-Poiseuille's equation (Suttera and Skalak, 1993) for the flow of two immiscible incompressible fluids as:

$Q_{w \mathrm{~m}}=\frac{\left(P_{w, \mathrm{~m}-1}-P_{w \mathrm{~m}}\right)}{\left(x_{m}-x_{m-1}\right)} \sum_{k=m}^{n} \lambda_{w k}$ 
Where $\lambda_{\alpha k}=\frac{\pi}{8 \mu_{\alpha}} R_{k}^{4}$ and $\mu_{\alpha}$ is the viscosity of the fluid in question. Take note that $\alpha$ corresponds to a fluid phase which can either be " $w$ " for water or " $o$ " for oil. The capillary pressure, $P_{c k}$, in the $k$ th tube, is given by:

$P_{c k}=P_{o k}-P_{w k}=\frac{2 \sigma \cos \theta}{R_{k}}$

Continuity of flow of incompressible fluids demands the total flow rate in region $m$ be equal to the injection rate, $Q_{t}$. That is,

$Q_{t}=Q_{w \mathrm{~m}}+Q_{o \mathrm{~m}}$

In terms of pressure drop, Equations (1) and (2) can be rewritten as:

$P_{w, \mathrm{~m}-1}-P_{w \mathrm{~m}}=\frac{Q_{w \mathrm{~m}}\left(x_{m}-x_{m-1}\right)}{\sum_{k=m}^{n} \lambda_{w k}}$

$P_{o, \mathrm{~m}-1}-P_{o \mathrm{~m}}=\frac{Q_{o \mathrm{~m}}\left(x_{m}-x_{m-1}\right)}{\sum_{k=1}^{m-1} \lambda_{o k}}$

With regard to studies of immiscible fluid displacement in a homogenous porous medium, fractional flow theory, developed by Buckley and Leverett (1942), plays a paramount role. The fractional flow of water, $f_{w}$, in region $m$ of the interacting tube-bundle model is obtained by subtracting Equation (6) from Equation (5), and substituting ( 3 ) and (4) in the resulting expression, while also recognizing that $f_{w}=Q_{w \mathrm{~m}} / Q_{t}$. It can be shown that:

$f_{w}=\frac{1+\frac{k_{o} A \Delta P_{c m}}{Q_{t} \mu_{o} \Delta x_{m}}}{1+\frac{k_{r o} \mu_{w}}{k_{r w} \mu_{o}}}$

Where $\Delta P_{c \mathrm{~m}}=P_{c \mathrm{k}}-P_{c \mathrm{k}-1}, \quad \Delta x_{m}=x_{m}-x_{m-1}$, and $m=k$. The absolute permeability, $k$, and permeabilities to oil and water,,$k_{o}$ and $k_{w}$, in region $m$ are given, respectively, as:

$k=\frac{\pi \sum_{k=1}^{n} R_{k}^{4}}{8 A}$

$k_{o}=\frac{\pi \sum_{k=1}^{m-1} R_{k}^{4}}{8 A}$

$k_{w}=\frac{\pi \sum_{k=m}^{n} R_{k}^{4}}{8 A}$
Where $A=\pi \sum_{k=1}^{n} R_{k}^{2}$ is the total cross section to flow. Hence, the relative permeabilities to oil and water in this region, $k_{r o}$ and $k_{r w}$, are:

$k_{\text {ro }}=\frac{\sum_{k=1}^{m-1} R_{k}^{4}}{\sum_{k=1}^{n} R_{k}^{4}}$

$$
k_{r w}=\frac{\sum_{k=m}^{n} R_{k}^{4}}{\sum_{k=1}^{n} R_{k}^{4}}
$$

The corresponding water saturation, $S_{w}$, is obtained from:

$S_{w}=\frac{\sum_{k=m}^{n} A_{k} L}{\sum_{k=1}^{n} A_{k} L}=\frac{\sum_{k=m}^{n} R_{k}^{2}}{\sum_{k=1}^{n} R_{k}^{2}}$

In terms of the injection rate, $Q_{t}$, it can be conveniently shown that the water flow rate, $Q_{w m}$, in region $m$ is given by:

$Q_{w \mathrm{~m}}=B_{m} Q_{t}-\frac{C_{m}}{x_{m}-x_{m-1}}$

Where:

$B_{m}=\frac{\sum_{k=m}^{n} \lambda_{w k}}{\sum_{k=1}^{m-1} \lambda_{o k}+\sum_{k=m}^{n} \lambda_{w k}}$

$C_{m}=\left(P_{c, \mathrm{~m}-1}-P_{c \mathrm{~m}}\right)\left(\frac{\sum_{k=1}^{m-1} \lambda_{o k} \cdot \sum_{k=m}^{n} \lambda_{w k}}{\sum_{k=1}^{m-1} \lambda_{o k}+\sum_{k=m}^{n} \lambda_{w k}}\right)$

Note that $\sum_{k=1}^{0} \lambda_{o k}=0$ and $\sum_{k=n+1}^{n} \lambda_{w k}=0$. As a result, $B_{1}=1, \quad C_{1}=0, B_{n+1}=0$ and $C_{n+1}=0$. This automatically implies that in the regions $m=1$ and $m=n+1, \quad Q_{w 1}=Q_{t}$ and $Q_{w, \mathrm{n}+1}=0$, respectively.

The viscous pressure drop in the water phase in region $m$ is obtained by substituting Equation (14) into Equation (5):

$P_{w, \mathrm{~m}-1}-P_{w \mathrm{~m}}=Q_{t}\left(\frac{B_{m}}{\sum_{k=m}^{n} \lambda_{w k}}\right)\left(x_{m}-x_{m-1}\right)-$

$-\frac{C_{m}}{\sum_{k=m}^{n} \lambda_{w k}}$ 
Note that in the region $m=1, P_{w, 0}=P_{i n}$, $P_{c, 0}=0$, and $x_{0}=0$; while in region $m=n+1$, $P_{w, \mathrm{n}+1}=P_{\text {out }}, P_{c, \mathrm{n}+1}=0$, and $x_{n+1}=L$.

Two kinds of boundary conditions, rate $\left(Q_{t}\right)$ and pressure $\left(P_{\text {in }}\right.$ and $\left.P_{\text {out }}\right)$, are possible in the operation of this model. The relationship between both boundary conditions is obtained from the summation of all pressure drop equations obtained from Equation (15) for $m=1,2, \ldots n+1$, as shown in Equation (16).

$$
\begin{aligned}
& P_{\text {in }}-P_{\text {out }}=Q_{t} \sum_{m=1}^{n+1} B_{m}\left(\frac{x_{m}-x_{m-1}}{\sum_{k=m}^{n} \lambda_{w k}}\right)- \\
& -\sum_{m=1}^{n+1}\left(\frac{C_{m}}{\sum_{k=m}^{n} \lambda_{w k}}\right)
\end{aligned}
$$

When two random boundary conditions are given, the third can be calculated based on Equation (16).

However, the evaluation of Equations (14), (15) and (16) depends compulsorily on the knowledge of the interface positions, $x^{\prime}$ s. It suffices to say the values of $x^{\prime}$ s reflect the state of the system at any point in time. The initial condition of flow is given by $x_{1 j}=x_{2 j}=\cdots=x_{n-1, j}=x_{n, j}=0$, corresponding to time $j \Delta t$ when $j=0$. At any other time $j \Delta t$, when $j=1,2,3, \ldots$, the interface positions, $x_{1 j}, x_{2 j}, \ldots, x_{n-1, j}$ and $x_{n, j}$, are determined. Thus, flow rate $\left(q_{k j}\right)$ in capillary $k$ at any time step $j>0$ is given by:

$q_{k j}=Q_{w k}-Q_{w k+1}$

Substituting $q_{k j}=A_{k} v_{k j}$ into Equation (17), where $A_{k}$ is the tube cross sectional area and $v_{k j}$ is the flow velocity in the tube, then:

$A_{k} v_{k j}=Q_{w k}-Q_{w k+1}$

It is recognized that $v_{k j}$ can be expressed in differential notation as $d x_{k j} / d t$. Therefore, Equation (18) becomes:

$A_{k} \frac{d x_{k j}}{d t}=Q_{w k}-Q_{w k+1}$
In finite (backward) difference form, Equation (19) can be expressed as:

$A_{k}\left(\frac{x_{k j}-x_{k j-1}}{\Delta t}\right)=Q_{w m}-Q_{w m+1}$

That can be rewritten as:

$$
\left(x_{k j}-x_{k j-1}\right) \frac{A_{k}}{\Delta t}+Q_{w \mathrm{~m}+1}-Q_{w \mathrm{~m}}=0
$$

where $k=m=1,2,3, \ldots, n$.

Upon substituting Equation (14) into Equation (21), the expression for tracking the displacement interfaces in the interacting tube bundle involving $n$ number of capillaries can be conveniently summarized as:

$$
\begin{aligned}
& \left(x_{k j}-x_{k j-1}\right) \frac{A_{k}}{\Delta t}+\frac{C_{m}}{x_{k j}-x_{k-1, j}}- \\
& -\frac{C_{m+1}}{x_{k+1 j}-x_{k j}}+\left(B_{m+1}-B_{m}\right) Q_{t}=0
\end{aligned}
$$

where $k=m=1,2,3, \ldots, n$, and $A_{k}=\pi R_{k}^{2}$.

\section{MECHANISM OF DISPLACEMENT}

The diagrammatic representation of the interacting tube bundle, shown in Figure 1, depicts all the flow regions and the interface positions when all the tubes have been invaded by the wetting phase. The figure also shows that the flow is faster in the smaller tubes and, hence, the oilwater interface travels further into the smaller tubes than into the larger tubes. This phenomenon is attributable to the assumption of pressure equilibration in the model. However, it is important to note that, at the start of the flow, the interface does not enter into all the tubes at the same time. Tube $n$ has the smallest radius and, consequently, the greatest capillary pressure. Therefore, displacement initially commences in this tube. When the interface in tube $n$ has travelled a distance $x_{n}^{*}$, at which point the non-wetting phase pressure, $P_{o 1}$, in tube $k=n$ falls just below the oilphase inlet pressure, $P_{o \text {,in }}$, in the next smallest tube $k=n-1$, the interface begins to advance in tube $n-1$.

A system of two capillary tubes can be used to illustrate the mechanism of interface entry, as shown in Figure 2. Initially, flow begins in the 


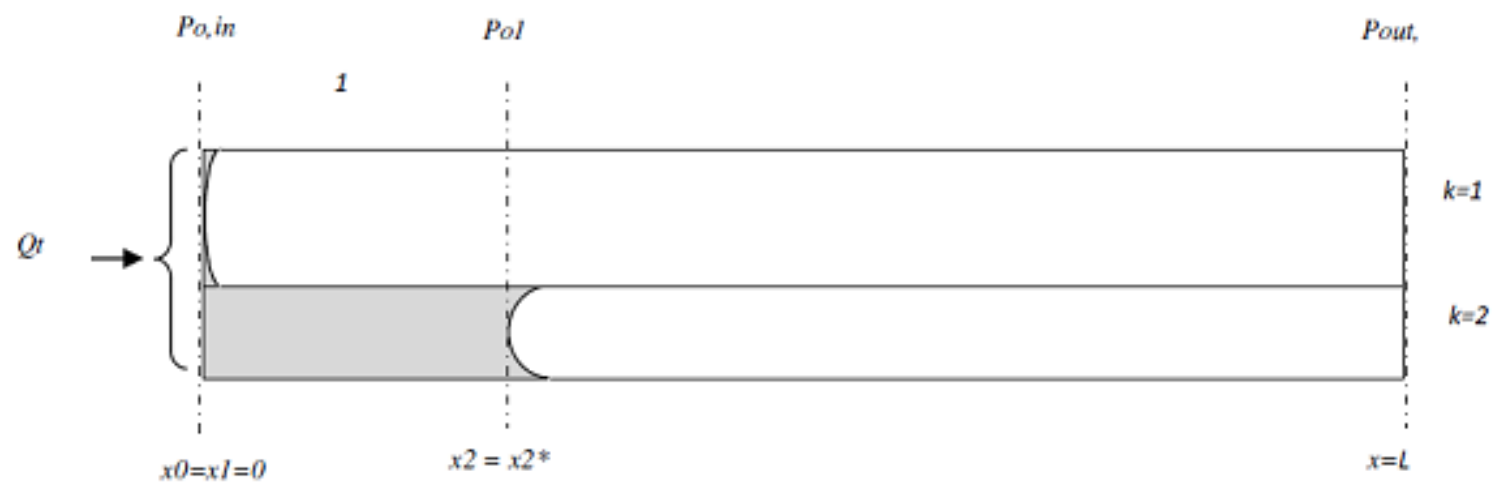

Figure 2. Mechanism of interface advancement in the tubes. An interface cups into the bigger tube when $\boldsymbol{P}_{o, \text { in }} \geq \boldsymbol{P}_{o 1}$.

smaller tube $k=2$. At the point where the interface reaches $x_{2}^{*}$, flow is just about to commence in tube $k=1$ as an interface cups into it at an oil-phase pressure of $P_{o, \text { in }}$. Mathematically, the interface begins to move in the second tube when:

$P_{o, \text { in }} \geq P_{o 1}$

Substituting $P_{o, \text { in }}=P_{i n}+P_{c 1}$ and $P_{o 1}=P_{w 1}+$ $P_{c 2}$ in the expression above produces:

$P_{\text {in }}-P_{w 1} \geq P_{c 2}-P_{c 1}$

The pressure drop $P_{i n}-P_{w 1}$ can be evaluated from Equation (15) as:

$P_{i n}-P_{w 1}=Q_{t}\left(\frac{x_{2}^{*}}{\lambda_{w 2}}\right)$

Substituting Equation (25) into (24), the critical interface position $x_{2}^{*}$ is calculated as:

$x_{2}^{*}=\frac{\left(P_{c 2}-P_{c 1}\right) \lambda_{w 2}}{Q_{t}}$

The time $t_{2}^{*}$ taken for the interface in tube $k=2$ to travel a distance $x_{2}^{*}$ can be obtained as follows:

$$
t_{2}^{*}=\frac{x_{2}^{*} A_{2}}{Q_{w 1}}
$$

Where $A_{2}$ is the area of tube $k=2$. Note that $Q_{w 1}=Q_{t}$, so:

$$
t_{2}^{*}=\frac{x_{2}^{*} A_{2}}{Q_{t}}
$$

In general, the condition for the interface to enter into any tube $k=1,2, \ldots, n-1$ is,

$P_{\text {in }}-P_{w 1}>P_{c, \mathrm{k}+1}-P_{c k}$

Substituting the correct pressure drop expression, $P_{i n}-P_{w 1}$, based on Equation (15), the critical interface position $x_{k+1}^{*}$ in tube $k+1$, where the interface in tube $k$ begins to advance, is:

$x_{k+1}^{*}=\frac{\left(P_{c, \mathrm{k}+1}-P_{c k}\right) \sum_{k+1}^{n} \lambda_{w, k+1}}{Q_{t}}$

The time $t_{k+1}^{*}$ taken for the interface in tube $k+1$ to travel a distance of $x_{k+1}^{*}$ can be obtained as follows:

$t_{k+1}^{*}=\frac{x_{k+1}^{*} A_{k+1}}{Q_{w 1}-Q_{w 2}}$

Where $A_{k+1}$ is the area of tube $k+1$. Recognizing that $Q_{w 1}=Q_{t}$, then:

$t_{k+1}^{*}=\frac{x_{k+1}^{*} A_{k+1}}{Q_{t}-Q_{w 2}}$

$Q_{w 2}$ is obtained from Equation (14) as:

$Q_{w 2}=B_{2} Q_{t}-\frac{C_{2}}{x_{2}-x_{k+1}^{*}}$

Whenever the condition given by Equation (28) is met in the displacement process, the wetting phase enters tube $k$. 


\section{SOLUTION OF THE MODEL}

The set of $n$ non-linear equations, given by Equation (22), describes how the system evolves over time. These equations can be expressed as the simultaneous zeroing of a set of $n$ functions of $n$ independent variables (Broyden, 1965). The functions are non-linear; their solutions cannot be easily obtained by simple mathematical operations. However, they can be recast in a form which makes them solvable by known methods of calculus. The solution approach adopted in this study is known as the method of linearization. The idea behind the method is to approximate the solution of the non-linear system of equations by the solution of a sequence of representative linear equations that is amenable to an infinite iterative process.

The initial condition of flow is given by $x_{1 j}=x_{2 j}=\cdots=x_{n-1}=x_{n j}=0$, corresponding to time $t=0$. The additional constraints necessary to compute the solution of Equations (17) are known as boundary conditions, $Q_{t}$ or $P_{\text {in }}$ and $P_{\text {out }}$. The relationship between the rate and the pressure boundary conditions is given by Equation (16).

Equation (22) can be rewritten as a set of $n$ functions given by:

$$
\begin{aligned}
& f_{k}\left(x_{1}, x_{2}, \ldots, x_{n}\right)=\left(x_{k j}-x_{k j-1}\right) \frac{A_{k}}{\Delta t}+ \\
& +\frac{C_{m}}{x_{k j}-x_{k-1, j}}-\frac{C_{m+1}}{x_{k+1 j}-x_{k j}}+ \\
& +\left(B_{m+1}-B_{m}\right) Q_{t}=0
\end{aligned}
$$

where $k=m=1,2,3, \ldots, n$.

From these $n$ functions $f_{k}\left(x_{1}, x_{2}, \ldots, x_{n}\right)$, $(k=1,2, \ldots, n)$ of $n$ variables, the positions of the interface, $x_{1}, x_{2}, \ldots, x_{n}$, are to be determined in such a way that $f_{1}=f_{2}=\ldots=f_{n}=0$. For every time step, the system is initialized at $\mathbf{x}=$ $\left(x_{1}, x_{2}, \ldots, x_{n}\right)^{T}$ and the vector $\mathbf{f}=\left(f_{1}, f_{2}, \ldots, f_{n}\right)^{T}$ is computed, where $f_{k}=f_{k}\left(x_{1}, x_{2}, \ldots, x_{n}\right)$, $(k=1,2, \ldots, n)$. At the start of the iteration in each time step, it is practical to set the vector $\mathbf{x}$ to the positions of the interface in the previous time step. The calculation then seeks a correction $\Delta \mathbf{x}$ such that for $k=1,2, \ldots, n$.
$f_{k}^{*}=f_{k}\left(x_{1}+\Delta x_{1}, x_{2}+\Delta x_{2}, \ldots, x_{n}+\Delta x_{n}\right)=0$

This set of functions can be expanded using the Taylor series to a first approximation as:

$f_{k}^{*}=f_{k}+\sum_{k=1}^{n} \frac{\partial f_{k}}{\partial x_{k}} \Delta x_{k}+\boldsymbol{O}\left(\Delta x_{k}\right)^{2}$

By neglecting the terms of order $\Delta x_{k}^{2}$ and higher, and setting $f_{k}^{*}=0$, a set of linear equations for the corrections $\Delta x_{k}, \quad(k=1,2, \ldots, n)$ that simultaneously move each function closer to zero is obtained.

$\sum_{k=1}^{n} \frac{\partial f_{k}}{\partial x_{k}} \Delta x_{k}+f_{k}=0, \quad k=1,2, \ldots, n$

This system for $\Delta x_{k},(k=1,2, \ldots, n)$ can be solved by Gaussian elimination. In vector form, Equation (35) can be written as:

$\mathbf{F} \Delta \mathbf{x}+\mathbf{f}=\mathbf{0}$

where $\mathbf{F} \equiv \frac{\partial \mathbf{f}}{\partial \mathbf{x}}$ is a Jacobian matrix which depends on $\mathbf{x}$. Once Equation (36) is solved, the corrected $\mathbf{x}+\Delta \mathbf{x}$ is again denoted by $\mathbf{x}$, and the process repeated with this new point until $\mathbf{f}$ is sufficiently small.

\section{ANALYSES AND DISCUSSION OF SIMULATION RESULTS}

The simulation model presented in this study is used to study the transport process in which water, representing the wetting phase, displaces oil, the non-wetting phase. The "porous medium" is a bundle of ten interacting uniform circular capillary tubes that follow a uniform pore size distribution $(50 \mu \mathrm{m} \leq R \leq 500 \mu \mathrm{m})$. The distance scale is rendered dimensionless so the maximum pseudolength of the model is unity. The model is initially filled with oil. In each simulation run, a constant rate boundary condition is imposed- water is injected at a constant rate at one end while oil is produced from the other end until the model is completely watered out. The intrinsic properties of the porous medium are reflected in its capillary pressure and relative permeability characterization, as shown graphically in Figures 3 and 4, respectively. While capillary-pressuresaturation data govern the pore size distribution of the medium, relative permeability reflects the ease of fluid transmission in the medium. 


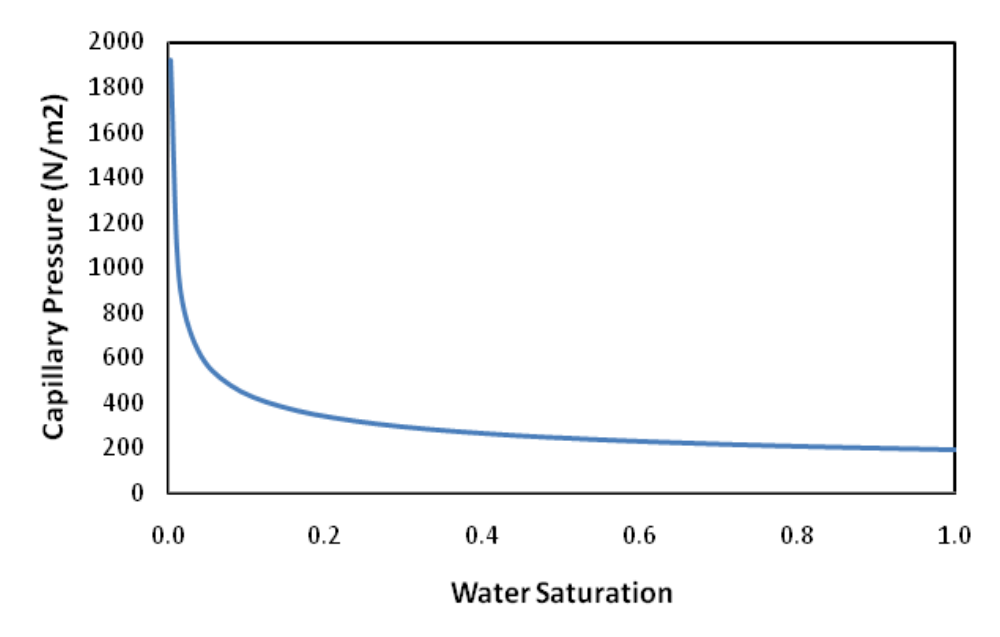

Figure 3. Capillary pressure curve for the model.

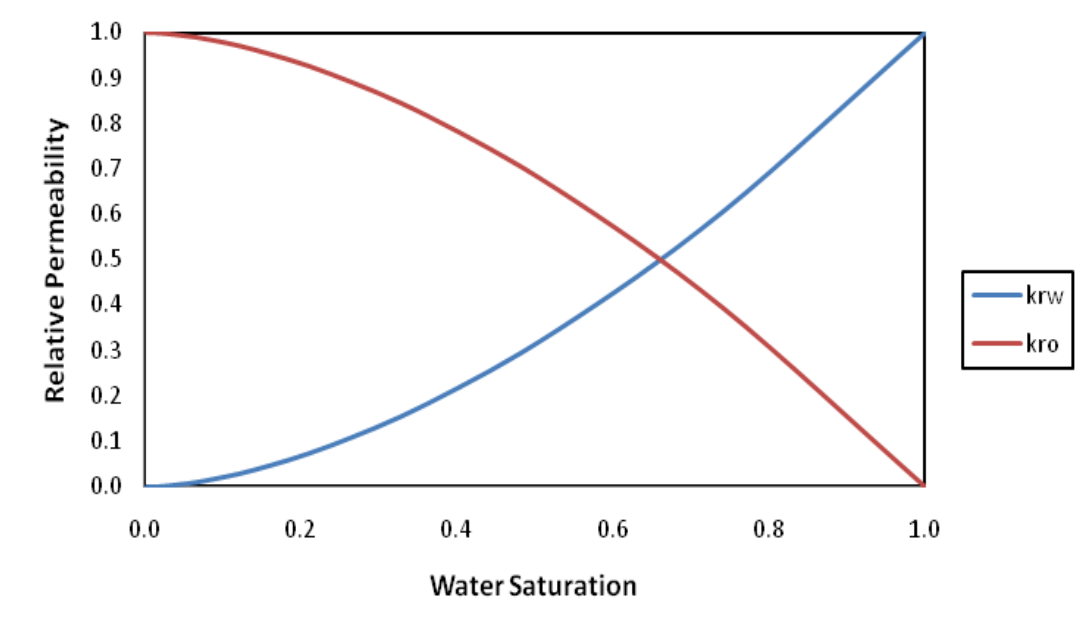

Figure 4. Relative permeability curves for the model.

The position of the fluid-fluid interface in each tube is tracked during the flow process. The results for various combinations of flow parameters including injection rate, viscosity ratio, interfacial tension, and wettability condition, are then analyzed and presented graphically in the form of saturation and interface profiles.

\subsection{Effect of injection rate}

Three different flow scenarios (Cases 1, 2 and 3) were run in order to investigate the influence of injection rate on flow behaviour. Table 1 reports the model parameters input for each run. The results are shown in Figures 5 through 10. The injected water first invades the smaller flow channels and travels a critical distance before permeating the bigger tubes. The critical distance depends significantly on the rate of injection and the mobility of the displacing fluid, as suggested by Equation (29).

At the low injection rate in Case 1, water advances into the smaller tubes first and has a delay in entering bigger tubes. Figure 5 shows the sequence of imbibition into the tubes. The low injection rate produces a flat, almost horizontal, saturation profile that keeps rising over time, as shown in Figure 6. In Case 2, the injection rate is higher. The implication of a higher rate is that the critical distance travelled by water in the smaller tubes is reduced. In other words, imbibition into the tubes is relatively rapid. Therefore, the profile for the displacement interface positions and the 
Table 1. Model parameters for investigating the effect of injection rate.

\begin{tabular}{lccc}
\hline \multicolumn{1}{c}{ Parameter } & \multicolumn{3}{c}{ Case } \\
& $\mathbf{1}$ & $\mathbf{2}$ & $\mathbf{3}$ \\
\hline Flow rate, $Q_{t}\left(\mathrm{~cm}^{3} / \mathrm{s}\right)$ & $1 \times 10^{-4}$ & $1 \times 10^{-3}$ & $1 \times 10^{-2}$ \\
\hline Oil viscosity, $\mu_{o}(\mathrm{~kg} / \mathrm{ms})$ & & 0.001 & \\
\hline Water viscosity, $\mu_{w}(\mathrm{~kg} / \mathrm{ms})$ & & 0.001 & \\
\hline Interfacial tension, $Q_{t}(\mathrm{~N} / \mathrm{m})$ & & 0.048 & \\
\hline Contact angle, $\theta(\mathrm{deg})$. & & 0 & \\
\hline
\end{tabular}

saturation evolution assume a steeper trend if compared to Case 1. This is shown in Figures 7 and 8. A further increase in the injection rate, as is the situation in Case 3, causes both the displacement interface and the saturation profiles to become even steeper; see flow behaviour shown in Figures 9 and 10 . The trends are in consonance with laboratory observations of immiscible displacement processes in real porous samples.

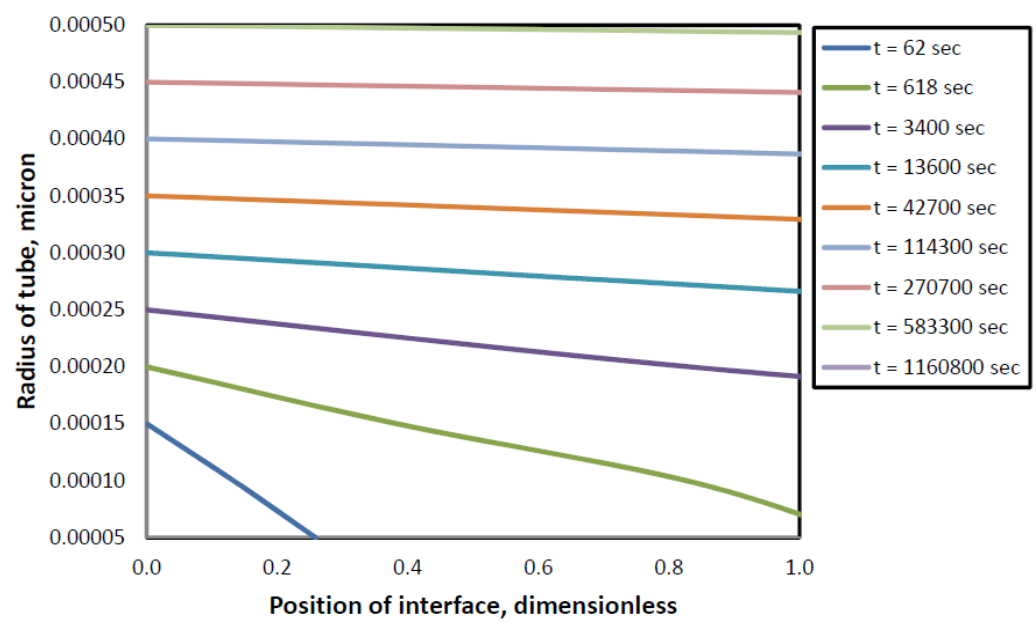

Figure 5. Interface profile, Case 1: $\boldsymbol{Q}_{\boldsymbol{t}}=\mathbf{0 . 0 0 0 1} \mathrm{cm}^{3} / \mathbf{s}$.

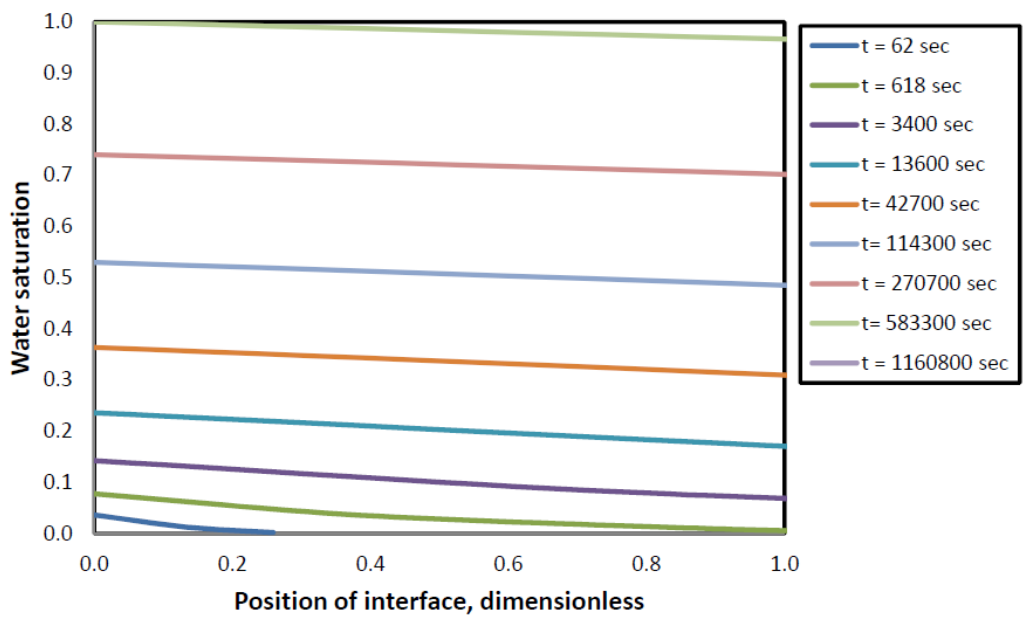

Figure 6. Saturation profile, Case 1: $Q_{t}=0.0001 \mathrm{~cm}^{3} / s$. 


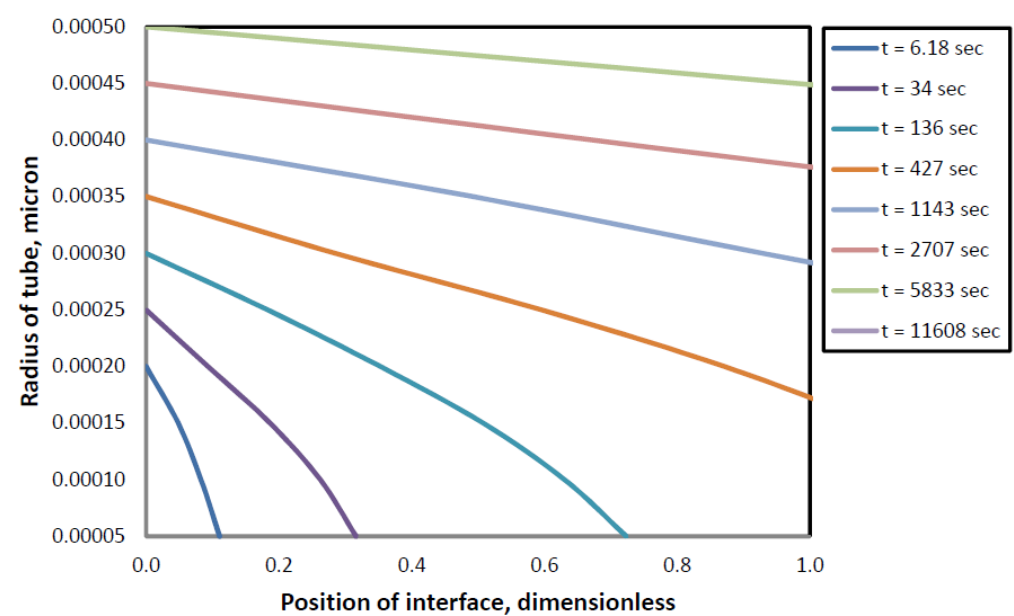

Figure 7. Interface profile, Case 2: $Q_{t}=\mathbf{0 . 0 0 1} \mathbf{c m}^{3} / s$.

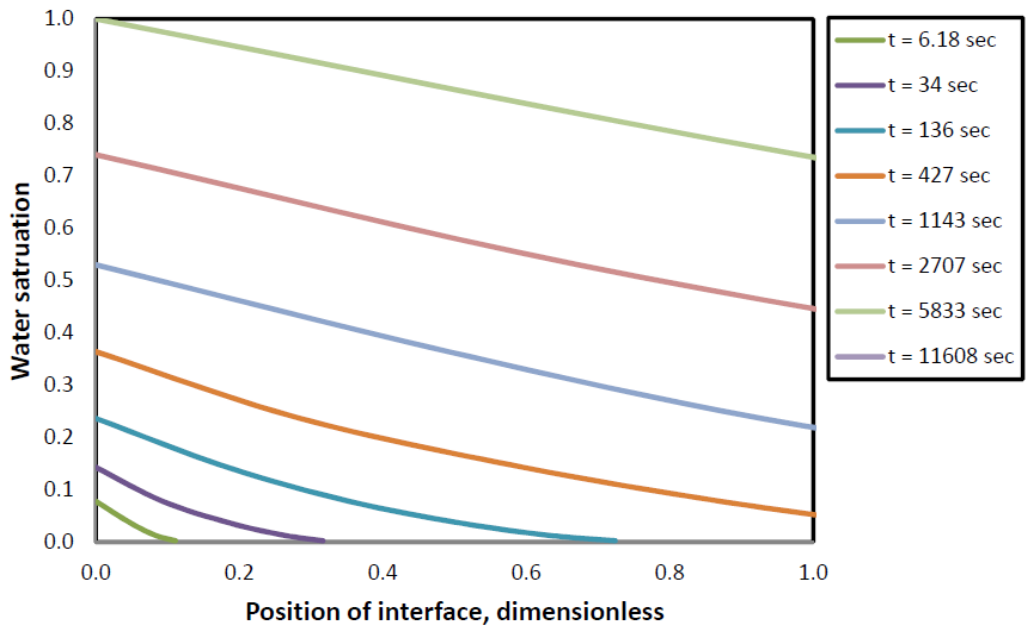

Figure 8. Saturation profile, Case 2: $Q_{t}=0.001 \mathrm{~cm}^{3} / \mathrm{s}$

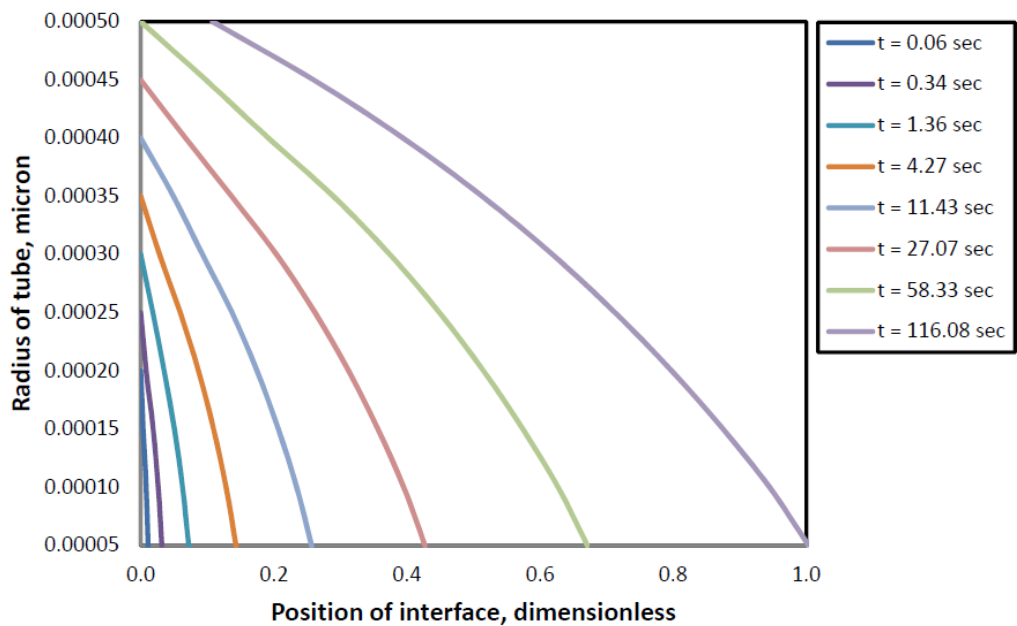

Figure 9. Interface profile, Case 3: $Q_{t}=0.01 \mathrm{~cm}^{3} / \mathbf{s}$. 


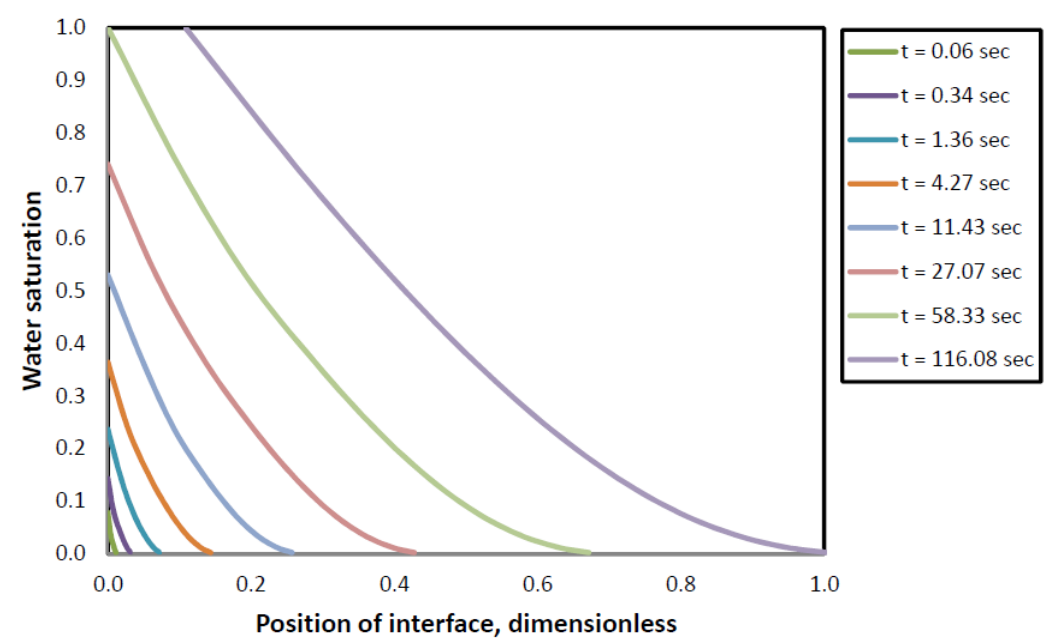

Figure 10. Saturation profile, Case 3: $Q_{t}=0.01 \mathrm{~cm}^{3} / \mathrm{s}$.

Table 2. Model parameters for investigating the effect of viscosity ratio.

\begin{tabular}{lccccc}
\hline \multicolumn{1}{c}{ Parameter } & \multicolumn{5}{c}{ Case } \\
& $\mathbf{4}$ & $\mathbf{5}$ & $\mathbf{6}$ & $\mathbf{7}$ & $\mathbf{8}$ \\
\hline Flow rate, $Q_{t}\left(\mathrm{~cm}^{3} / \mathrm{s}\right)$ & & \multicolumn{1}{c}{$1 \times 10^{-2}$} \\
Oil viscosity, $\mu_{o}(\mathrm{~kg} / \mathrm{ms})$ & 0.001 & 0.001 & 0.001 & 0.005 & 0.010 \\
Water viscosity, $\mu_{w}(\mathrm{~kg} / \mathrm{ms})$ & 0.010 & 0.005 & 0.001 & 0.001 & 0.001 \\
& & & & & \\
Viscosity ratio, $($ dimensionless $)$ & 0.1 & 0.2 & 1.0 & 5.0 & 10.0 \\
Interfacial tension, $Q_{t}(\mathrm{~N} / \mathrm{m})$ & & & 0.048 & & \\
Contact angle, $\theta($ deg. $)$ & & & 0 & & \\
\hline
\end{tabular}

\subsection{Effect of viscosity ratio}

A second set of runs compares the results obtained for five different viscosity ratios. Viscosity ratio, as used herein, refers the quotient of the viscosity of displaced phase to that of the displacing phase. Table 2 shows, at a glance, the combination of parameters used to demonstrate the effect of viscosity ratio on the flow behaviour. The results of the displacement are as shown in Figures 11 and 12 when $26 \%$ pore volume of water has been injected into the model. It is clear from the observation of saturation profiles, Figure 11, that the overall displacement is nearly piston-like at low viscosity ratios of 0.1 and 0.2 . As the viscosity ratio increases to 1.0 , the saturation profile assumes a relatively gentler slope. A further increase of viscosity ratio to 5.0 and 10.0 produces a flattening of the saturation profile. This flow behaviour is understandable from a consideration of the interface positions in the capillary tubes, as shown in Figure 12. At low viscosity ratios, the displacing phase is more viscous and less mobile; hence, it is less ready to penetrate the narrower tubes.

These low viscous ratios cause the fluid-fluid interfaces in the capillary tubes to travel closely together, thereby producing a near "step-function" saturation profile. As the viscosity ratio increases to 1.0, the transport ability of the displacing phase improves slightly. Therefore, the interfaces travel fairly separated such that those in the smaller tubes are significantly ahead of those in the wider tubes. This separation creates a less steep saturation profile. As the viscosity ratio further increases beyond 1.0, the displacing phase becomes less viscous and more mobile than the 


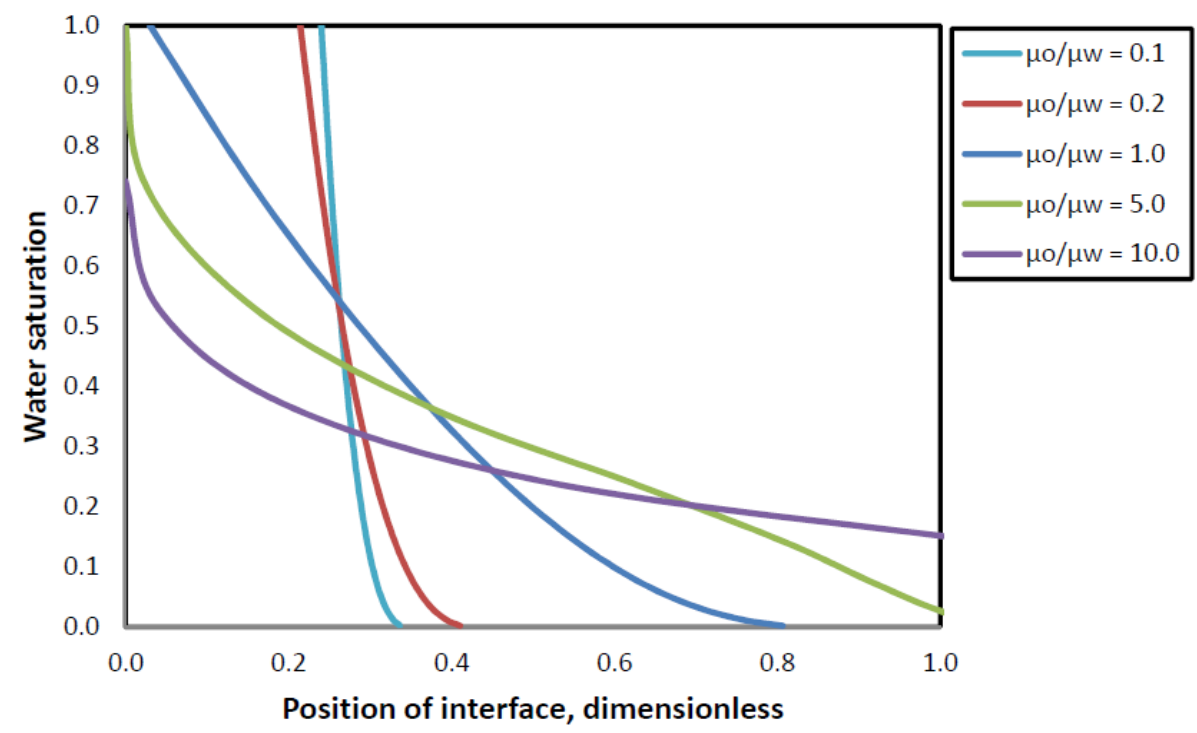

Figure 11. Saturation profiles at different viscosity ratios at $26 \%$ pore volume injection.

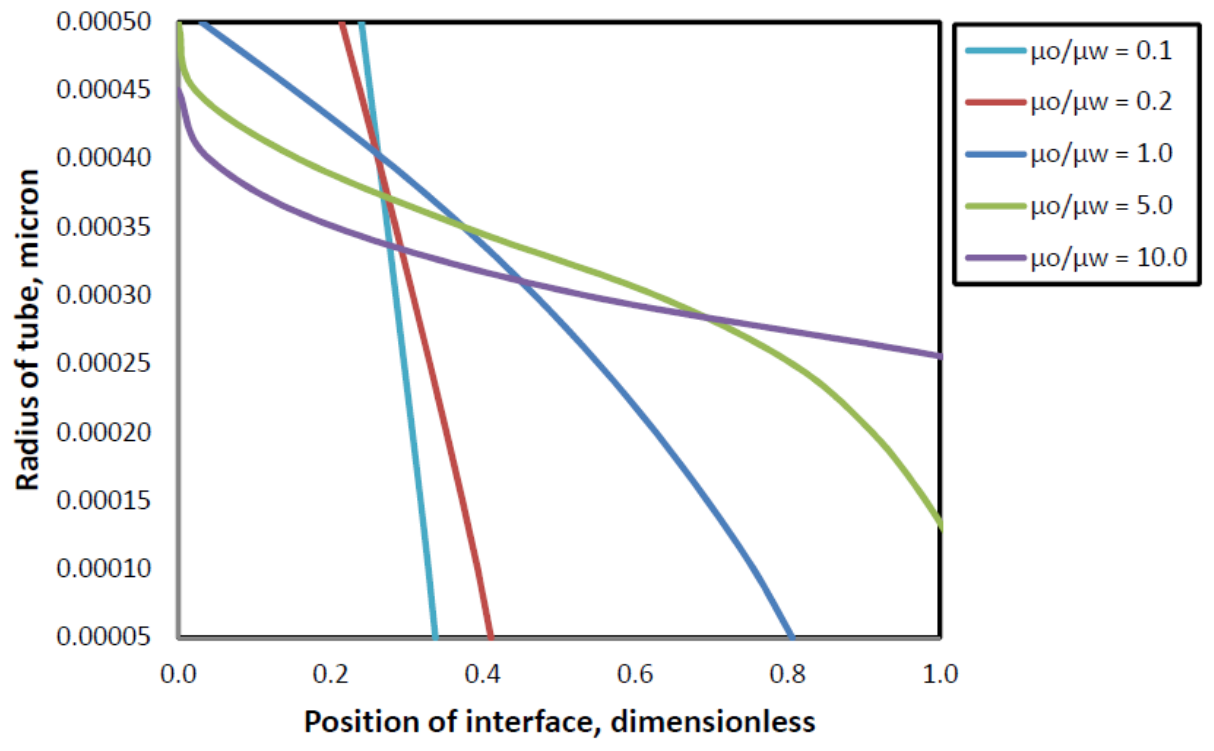

Figure 12. Interface profile at different viscosity ratios.

displaced phase. The tendency for the displacing phase to invade the smaller tubes is enhanced considerably. This invasion is marked by the dramatic flattening of the saturation profile and the displacement interface profile depicting that the fluid-fluid interfaces travel further in the smaller tubes than in the wider tubes. This phenomenon is identical to viscous fingering as observed in immiscible displacement processes at high viscosity ratios in real porous media.

\subsection{Effect of interfacial tension}

In order to study the effect of interfacial tension, the model was run for five different cases, as shown in Table 3. In each case, all flow parameters are kept constant except for the interfacial tension which is varied between $0.048 \mathrm{~N} / \mathrm{m}$ and $0.0001 \mathrm{~N} / \mathrm{m}$. The results are shown in Figure 13, at time $100 \mathrm{~s}$, when $33 \%$ pore volume of water has been injected into the model. It is observed that a reduction in the interfacial tension steepens the saturation profile, thereby enhancing the oil recovery. The reason for this improvement is 
Table 3. Model parameters for investigating the effect of interfacial tension.

\begin{tabular}{lccccc}
\hline \multicolumn{1}{c}{ Parameter } & $\mathbf{5}$ & \multicolumn{5}{c}{ Case } \\
& $\mathbf{9}$ & $\mathbf{1 0}$ & $\mathbf{1 1}$ & $\mathbf{1 2}$ & $\mathbf{1 3}$ \\
& & & & & \\
\hline Flow rate, $Q_{t}\left(\mathrm{~cm}^{3} / \mathrm{s}\right)$ & & & $1 \times 10^{-2}$ \\
Oil viscosity, $\mu_{o}(\mathrm{~kg} / \mathrm{ms})$ & & & 0.001 \\
Water viscosity, $\mu_{w}(\mathrm{~kg} / \mathrm{ms})$ & & & 0.001 & \\
Interfacial tension, $Q_{t}(\mathrm{~N} / \mathrm{m})$ & 0.048 & 0.01 & 0.005 & 0.001 & 0.0001 \\
Contact angle, $\theta(\mathrm{deg})$. & & \multicolumn{4}{c}{0} \\
\hline
\end{tabular}

explainable by observing the positions of the displacement interfaces in the capillary tubes, as shown in Figure 14. As the interfacial tension reduces, the sweep efficiency of the displacing phase improves. This results in a piston-like displacement such that the interfaces in the tubes travel closer together. In other words, reducing the interfacial tension increases the mobility of the displaced fluid. This is the idea behind enhancing oil recovery from petroleum reservoirs by using surfactants to reduce oil-water interfacial tension.

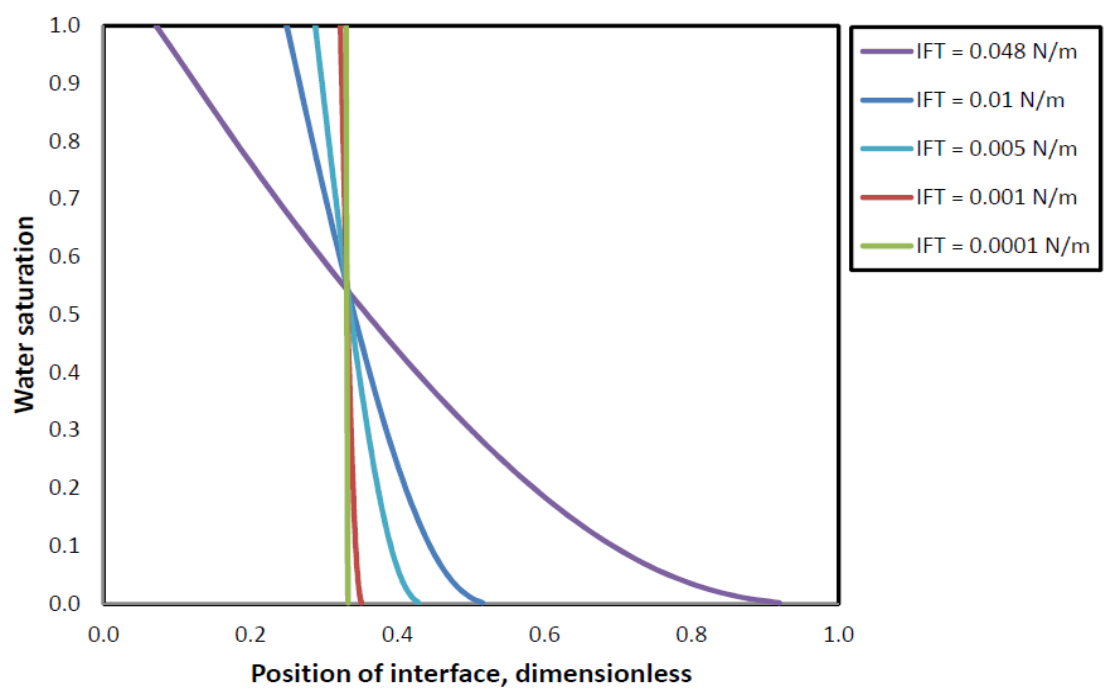

Figure 13. Saturation profiles at different values of interfacial tension at $33 \%$ pore volume injection.

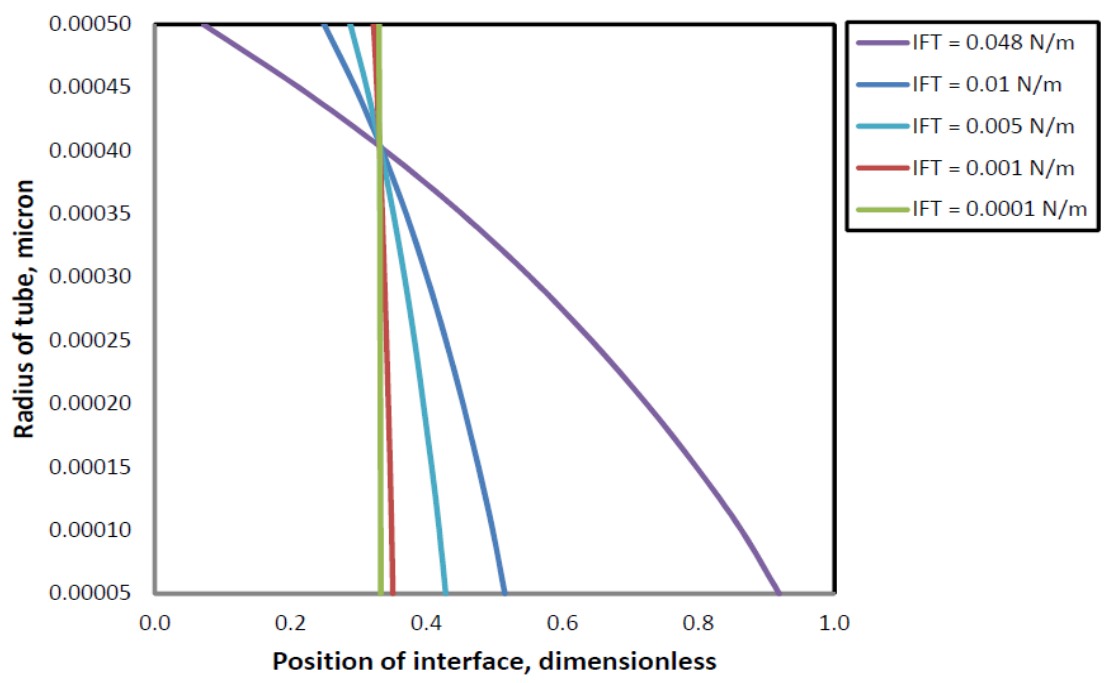

Figure 14. Interface profile at different values of interfacial tension at 33\% pore volume injection. 
Table 4. Model parameters for investigating the effect of wettability.

\begin{tabular}{lccccc}
\multicolumn{1}{c}{ Parameter } & \multicolumn{5}{c}{ Case } \\
& $\mathbf{1 4}$ & $\mathbf{1 5}$ & $\mathbf{1 6}$ & $\mathbf{1 7}$ & $\mathbf{1 8}$ \\
\hline & & & & & \\
& & & $1 \times 10^{-2}$ & & \\
Flow rate, $Q_{t}\left(\mathrm{~cm}^{3} / \mathrm{s}\right)$ & & & 0.001 & & \\
Oil viscosity, $\mu_{o}(\mathrm{~kg} / \mathrm{ms})$ & & & 0.001 & & \\
Water viscosity, $\mu_{w}(\mathrm{~kg} / \mathrm{ms})$ & & & 0.048 & & \\
Interfacial tension, $Q_{t}(\mathrm{~N} / \mathrm{m})$ & 0 & 45 & 60 & 75 & 90 \\
Contact angle, $\theta$ (deg. $)$ & & & & &
\end{tabular}

It is also evident from Figures 13 and 14 that very low values of interfacial tension cause the two fluids to behave as though they are hydraulically connected, in which case the capillary pressure across the interface of separation is infinitesimally small. Owing to the fact that there is transfer flow between the flow channels, closely related flow paths (i.e. tubes having little differences in their dimensions) behave as one flow channel. The implication of this phenomenon of "capillary lumping" is that at ultra low interfacial tension, the entire tube system mimics a single flow conduit and, thus, the displacement is far more piston-like.

\subsection{Effect of wettability}

The effect of wettability on the saturation profile and the interface profile is investigated in five different runs. In each run, the wettability condition is reflected in the contact angle, indicating the inclination of the fluid-fluid interface to the walls of the porous structure measured through the wetting phase, Figure 15 . The contact

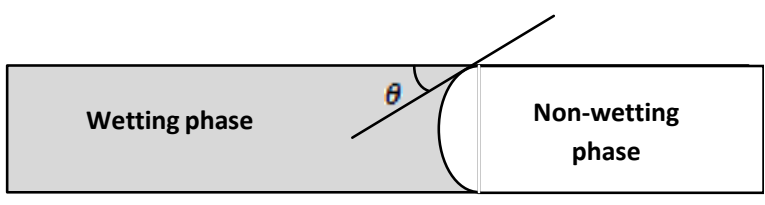

Figure 15. Measurement of contact angle, $\boldsymbol{\theta}$.

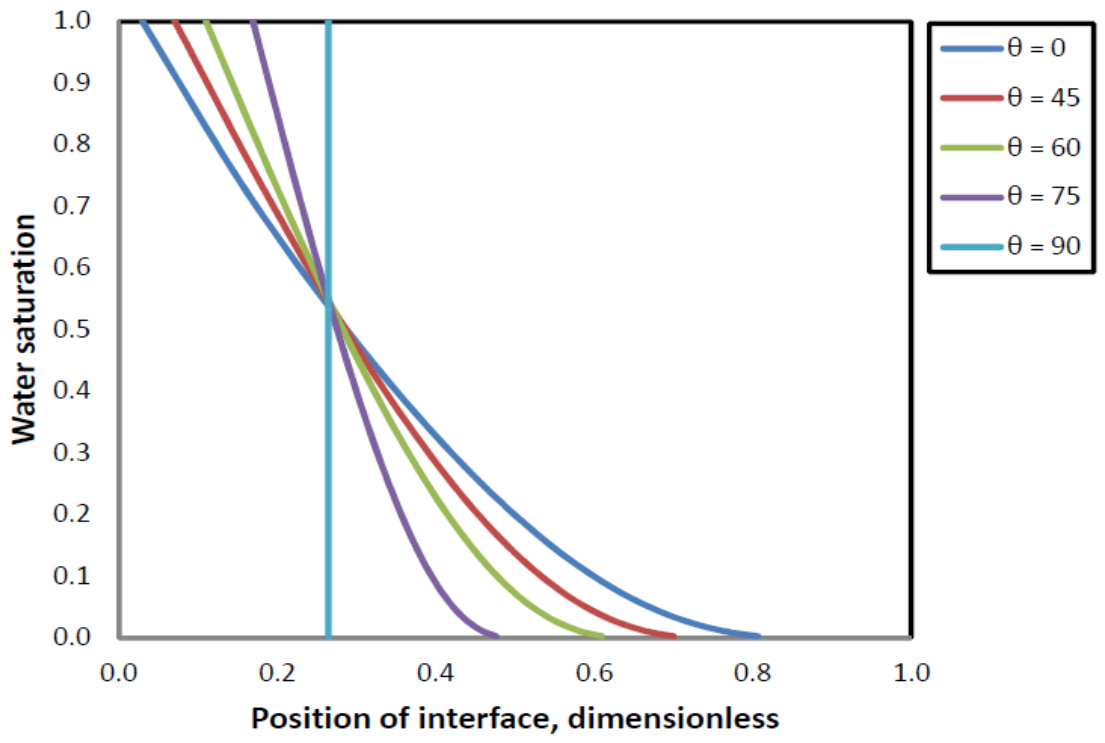

Figure 16. Saturation profiles at different wettability conditions (contact angles) at 26\% pore volume injection. 


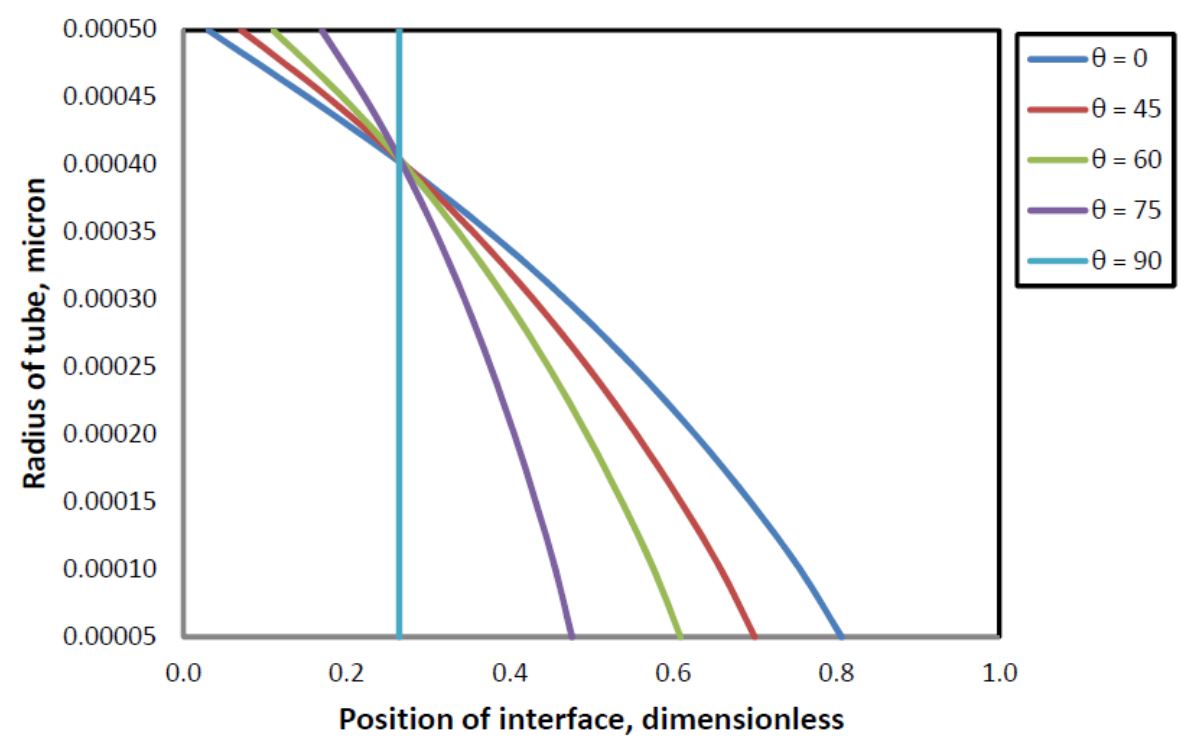

Figure 17. Interface profile at different wettabilitity conditions (contact angles) at $26 \%$ pore volume injection.

angle is varied between $0^{o}$ (perfect wetting condition for the displacing phase) and $90^{\circ}$ (intermediate wetting condition for both phases), and the results are reported at the point where $26 \%$ pore volume of the displacing phase has been injected. Table 4 reports the combination of flow parameters.

At $0^{\circ}$ contact angle, the displacing phase perfectly wets the walls of the medium. This is marked by the ease of advance of the displacing phase in the narrow pores of the medium. Thus, the displacing phase travels distinctively further in the narrower tubes than in the wider tubes (Figures 16 and 17). With increasing contact angle, the displacing phase gradually loses its wetting ability to the displaced phase. This produces a readjustment of the interface positions in the tubes as the displacing phase propagates less readily in the narrower channels. Hence, the saturation and the interface profiles steepen. A further increase of the contact angle to $90^{\circ}$ results in a wetting condition in which both the displacing and the displaced fluids have equal affinity for the walls of the medium. In this condition, the fluid-fluid interface is perpendicular to the walls of the medium and no capillary pressure acts across the interface. Due to transfer flow across flow channels, the entire tube system behaves as a single flow conduit. The overall effect is a displacement which is absolutely piston-like and is shown in the saturation and interface profiles.

\section{CONCLUSIONS}

This study puts forward a new mathematical model that seeks to further investigate multiphase flow in porous media. The flow medium considered in this work is a representative "physical model" made up of interacting parallel uniform circular capillary tubes, assembled to form a porous structure.

The concluding points, drawn from this study, are summarized below:

1. The derivation method pursued in this work furnishes a more tractable model than the model proposed by Dong et al. $(2005,2006)$. It is important to point out that Dong et al. developed their model based upon consideration of pressure drop terms in the bundle of tubes. The downside of their model is that each of the resulting $n$ evolution equations contains $n$ unknown interface positions, where $n$ corresponds to the number of tubes comprising the porous structure. A greater computational resource is unavoidably required in determining the unknowns. This present study proposes a model which takes into consideration flow rate terms and arrives at relatively simpler $n$ evolution equations, each of which contains only three unknowns and, as a result, calls for a shorter computational time. 
2. The model correctly replicates observable behaviours of multiphase flow in real porous media. With the inclusion of flow dependence between adjacent flow channels, the model predicts that the wetting-phase and displacing fluid imbibes first into smaller flow channels and also travels further into the smaller flow channels rather than in the larger ones.

3. The trends observed in the analysis of the simulation results, furnished by the proposed model, are reasonable and qualitatively correct for all combinations of parameters and important factors, such as injection rate, viscosity ratio, interfacial tension, and wettability condition, affecting immiscible transport processes in porous media.

4. Although irreducible wetting-phase saturation and residual wetting-phase saturation are not taken into consideration in the present study, the model can be used to investigate the dynamics of flow between the two saturation levels.

\section{ACKNOWLEDGEMENTS}

The authors gratefully acknowledge the financial support of Mobil Producing Nigeria Unlimited (MPN), a subsidiary of Exxon Mobil Corporation.

\section{REFERENCES}

Bartley, J. R.; Ruth, D. W. Relative permeability analysis of tube bundle models. Transport in Porous Media, v.36, p. 161-187, 1999. http://dx.doi.org/10.1023/A:1006575231732

Bartley, J. R.; Ruth, D. W. Relative permeability analysis of tube bundle models, including capillary pressure. Transport in Porous Media, v. 45, p. 447480, 1999.

Blunt, M.; King, P. Relative permeabilities from two- and three-dimensional pore-scale network models. Transport in Porous Media, v. 6, p. 407433, 1991. http://dx.doi.org/10.1007/BF00136349
Brakel, J. V. Pore space models for transport phenomena in porous media, review and evaluation with special emphasis on capillary liquid transport. Powder Technology, v. 11, p. $205-236$, 1975.

Broyden, C. G. A class of methods for solving non-linear simultaneous equations. Mathematics of Computation AMS, v. 19, p. 577-593, 1965. http://dx.doi.org/10.1090/S0025-5718-1965-0198670-6

Buckley, S. E.; Leverett, M. C. Mechanism of fluid displacement in sands. Petroleum Transactions AIME, v. 146, p. 107-116, 1942.

Carman, P. C. Fluid Flow through granular beds. Transactions IChemE, v. 15, p. 150-166, 1937.

Carman, P. C. Flow of gases through porous media. London: Butterworths, 1956.

Dias, M.; Payatakes, A. Network models for twophase flow in porous media, Part 1: Immiscible microdisplacement of non-wetting fluids. Journal of Fluid Mechanics, v. 164, p. 305-336, 1986. http://dx.doi.org/10.1017/S0022112086002574

Dong, M.; Dullein, F. A. L.; Zhou, J. Characterization of waterflood saturation profile histories by the 'complete' capillary number. Transport in Porous Media, v. 31, p. 213-237, 1998. http://dx.doi.org/10.1023/A:1006565621860

Dong, M.; Dullein, F. A. L.; Dai, L.; Li, D. Immiscible displacement in the Interacting capillary bundle model, Part 1: Development of interacting capillary bundle model. Transport in Porous Media, v. 59, p. 1-18, 2005. http://dx.doi.org/10.1007/s11242-004-0763-5

Dong, M.; Dullein, F. A. L.; Dai, L.; Li, D. Immiscible displacement in the interacting capillary bundle model, Part 2: Applications of model and comparison of interacting and non-interacting capillary bundle models. Transport in Porous Media, v. 63, p. 289-304, 2006.

http://dx.doi.org/10.1007/s11242-005-6530-4

Dullein, F. A. L. New network permeability model of porous media. American Institute of Chemical Engineers Journal, v. 21(2), p. 299-307, 1975. http://dx.doi.org/10.1002/aic.690210211

Fatt, I.; Dykstra, H. Relative permeability studies. Petroleum Transactions AIME, v. 192, p. 249-256, 1951. 
Fatt, I. The network model of porous media I: Capillary pressure characteristics. Petroleum Transactions AIME, v. 207, p. 144-159, 1956.

Fatt, I. The network model of porous media II: Dynamic properties of a single size tube network. Petroleum Transactions AIME, v. 207, p. 160-163, 1956.

Fatt, I. The network model of porous media III: Dynamic properties of networks with tube radius distribution. Petroleum Transactions AIME, v. 207, p. 164-181, 1956.

Gates, J. I.; Leitz, W. T. Relative permeabilities of California cores by the capillary - pressure method. Proceedings API Meeting, Los Angeles, California, May 11, 1950.

Kozeny, J. Ueber kapillare leitung des wassers im boden. Sitzungsber Akad. Wiss., Wien, v. 136 (2A), p. 271-306, 1927.

$\varnothing$ ren, P. E.; Bakke, S.; Arntzen, O. J. Extending predictive capabilities to network models. SPE Journal, v. 3, p. 324-336, 1998.
Patzek, T. W. Verification of a complete pore network simulator of drainage and imbibitions. SPE Journal, v. 6(2), p. 144-156, 2001. http://dx.doi.org/10.2118/71310-PA

Purcell, W.R. Capillary pressures - their measurement using mercury and the calculation of permeability. Petroleum Transactions AIME, v. 186, p. 39-48, 1949.

Rapoport, L. A.; Leas, W. J. Relative permeability to liquid in liquid-gas systems. Petroleum Transactions AIME, v. 192, p. 83-98, 1951.

Rothfeld, L. B. Gaseous counterdiffusion in catalyst pellets. American Institute of Chemical Engineers Journal, v. 9, p. 19-24, 1963. http://dx.doi.org/10.1002/aic.690090105

Sutera, S. P.; Skalak, R. The history of Poiseuille's law. Annual Review of Fluid Mechanics, v. 25, p. 1-20, 1993.

http://dx.doi.org/10.1146/annurev.fl.25.010193.000245 